Espacio, Tiempo y Forma, Serie NV, H. Moderna, t. 15, 2002, págs. 11-34

\title{
Natalidad, nupcialidad y fecundidad en Mérida durante el siglo XVI
}

\author{
José Antonio Ballesteros díez *
}

\section{RESUMEN}

Este articulo es un estudio demográfico de Mérida durante el siglo Xvi, que ha sido realizado a partir de las actas de bautismo, confirmación

y matrimonio, por el procedimiento de la agrupación de familias, o método francés, analizando la natalidad con detalle de partos simples y dobles,

las concepciones, las distribuciones temporales y sus tendencias, se ha determinado en número de familias y sus composiciones, obteniendo el coeficiente para la transformación de vecinos cabezas de familia en habitantes, tanto el general de la ciudad como los correspondientes a las comunidades cristiana y morisca;

se han determinado las edades de acceso al matrimonio, los intervalos genésicos en las mujeres, los estados prenupciales, la distribución temporal de la nupcialidad y los grupos de ilegítimos, expósitos, esclavos y moriscos.

\section{ABSTRACT}

This is a demographic study of Mérida (a town of Castilla, Spain) during the $x v$ ! century, from fhe baptism, confirmation and marriages registers, according to the process of the association of families by analysing the natality in detail of simple and double childbirths, and the concepcions, the temporal distributions and their tendencies; with the determination of the number of families and their composition, by obtaining the coeffcient in order to transform the neighbours into inhabitants. Both the general one of the town and the one wich belongs to the christian and moorish communities; the age of acces to marriage, genesis inervals in women, prenuptial states, temporal distribution of nupcitiality, illegitimate groves, abandoned people, slaves and moorishes have been determined

* UNED. 


\section{INTRODUCCIÓN}

Para el estudio de la población emeritense durante el siglo xvi hemos dispuesto de los libros de las parroquias de Santa María la Mayor (hoy concatedral en el arzobispado de Mérida-Badajoz) y Santa Olalla, únicas existentes en aquella centuria, que estos libros no cubren en su totalidad, y también presentan discontinuidades entre unos y otros; además, intuimos que las inscripciones se hacían en hojas, o pliegos sueltos, que luego se encuadernaban, es decir, los libros son posteriores, en algunos casos con muchos años de diferencia, a la formulación del registro por escrito del acta sacramental; en ninguna de las dos parroquias se tienen libros de fallecidos en este siglo. La cronología de los registros es la siguiente:

- Parroquia de Santa María

- Libro 1 de Bautizados, de 1526 a 1593

- Libro 2 de bautizados, de 1558 a 1593

- Libro 3 de bautizados, de 1593 a 1621

- Libro 1 de casados y velados, de 1594 a 1624

- Parroquia de Santa Olalla

- Libro 1 de bautizados, de 1581 a 1633

- Libro 1 de casados y velados, de 1595 a 1661

Es decir, para la primera mitad de la centuria solamente disponemos de apenas 13 años de registros bautismales en la parroquia de Santa María, luego hay un vacío desde 1539 a 1558, y para las dos décadas finales contamos con las inscripciones de las dos parroquias.

Como nuestro propósito final es hacer el estudio demográfico de Mérida por el procedimiento de la agrupación de familias, al no disponer de datos en continuidad a todo lo largo del siglo, y carecer también de los registros de fallecidos, hemos completado parcialmente la información necesaria cruzando los datos de ambos tipos de registros y contando también con las actas de las ceremonias de confirmación, incluidas en los libros bautismales, de las que no había ninguna referencia, y que corresponden a celebraciones conjuntas para las dos parroquias que se realizaron en las fechas siguientes:

CONFIRMACIONES:

- 13 de agosto de 1564

- 20 de septiembre de 1582

- 17 de marzo de 1583

- 8 de enero de 1587

- 17 de mayo de 1587 
En estas actas se relacionan a los confirmados por su nombre de pila, solamente, indicándose los nombres y apellidos de los padres, igual que se hacía en los registros bautismales.

La clasificación y ordenación de los datos procedentes de estas fuentes nos ha puesto en presencia del problema de la filiación, pues hay personas que llevaban el apellido del padre, otras el de la madre, y un tercer grupo es el de aquellos cuyo apellido no coincide con el de ninguno de sus progenitores, y además dificultado por el hecho de que la gran mayoría de la población solamente usase un apellido. Al hacer la agrupación por familias aparecía aun más agravado este problema de la filiación, porque dentro de una misma familia es frecuente que haya hijos con filiación paterna, otros materna e, incluso, algunos con apellido diferente al de los padres. Para cuantificar estas circunstancias, y tomando como base los libros matrimoniales, en los que se registran los contrayentes con su nombre y apellido, así como los de sus progenitores respectivos, también con nombre y apellido, y sin contar aquellos matrimonios en que ambos cónyuges tienen el mismo apellido y es el que llevan sus hijos, el resultado es el siguiente:

FILIACIONES:

- Apellido del padre: $602 \quad$ < $\quad 39,7 \%$

- Apellido de la madre: $354 \quad$ < $\quad 23,4 \%$

- Apellido distinto: $559 \quad<\quad 36,9 \%$

distribución que exponemos gráficamente, para la mejor visualización del problema.

\section{FILIACIONES}

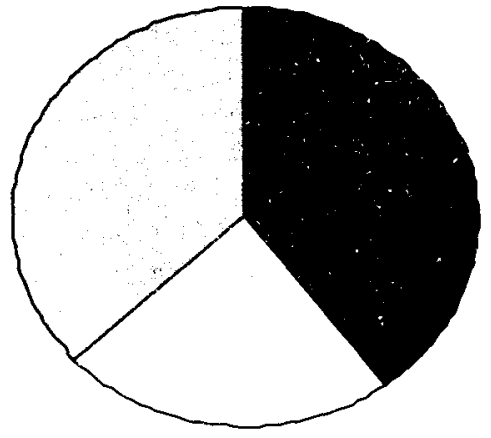

A pellido paterno

$\square$ A pellido materno

Otro apellido 
Para completar las informaciones que aportaban cada tipo de registros, se han tenido en cuenta también las filiaciones de los padrinos cuando se detallaba que formaban matrimonio, y en muchos casos, en ausencia de un segundo apellido, las profesiones u oficios de los distintos participantes, que no siempre se expresaba.

En los libros matrimoniales se registran dos ceremonias diferentes referidas a cada unión: la de los esponsales, que muchas veces no se realizaba en el templo parroquial sino en alguna casa, bien de la familia, de los contrayentes o de un tercero; y la ceremonia de las velaciones, que siempre era en la iglesia, aunque en algunas ocasiones las dos se celebraban conjuntamente; o bien una en una parroquia y la otra en otra, e incluso en una localidad distinta. Todo esto nos ha llevado a la unificación de los enlaces matrimoniales, computando solamente una de las ceremonias y tomando como referencia temporal la fecha de la primera. La cuantificación de los registros matrimoniales, tal como hemos dicho, nos ha llevado a comprobar cómo algunos, que han hecho estudios sobre estos libros por el procedimiento simple de los recuentos, casi han duplicado el número de uniones matrimoniales producidas con respecto a las que realmente se han producido.

Igualmente, tras la lectura de los registros bautismales, y para hacer la transposición de bautismos a nacimientos, se han eliminado de éstos los correspondientes a los bautismos de adultos, generalmente esclavos, de modo que el número de nacimientos computados es menor que el de bautismos; lo cual también ha sido un error, que hemos comprobado, han tenido los que han tomado como iguales el número de bautismos y el de nacimientos, al haberse limitado a contar los registros sin leer completamente el texto de los mismos.

En el presente trabajo vamos a realizar un análisis cuantitativo de los datos recogidos, pues aunque la lectura completa de los registros proporciona mucha información, especialmente para la historia social ${ }^{1}$, aquí nos vamos a centrar en la determinación de algunos valores demográficos de Mérida durante el siglo XVI.

\section{PRIMERA MITAD DEL SIGLO XVI}

Como ya se ha indicado, para esta etapa sólo se dispone de un libro de bautismos, el de la parroquia de Santa María, que registra las cere-

\footnotetext{
Ballesteros Diez, J. A., "Bautismos e historia social en Mérida a principios del siglo XVI». Revista de Estudios Extremeños, Tomo LVII, Núm. Ii, págs. 647 a 659. Badajoz, 2001. “Bautismos, confirmaciones y matrimonios en la historia social de Mérida en la segunda mitad del siglo xVı". Revista de Estudios Extremeños, Tomo LVIII, Núm. III, págs. 941 a 989. Badajoz, 2002.
} 
monias bautismales celebradas entre el mes de Junio del año 1526 y el de Julio de 1539, pero aunque en su totalidad recoge trece años, para la determinación de algunos valores y la comparación con otros registros parroquiales, solamente consideramos los datos correspondientes a las doce anualidades completas, es decir, las que van desde 1527 hasta 1538 , ambas incluidas; los bautismos registrados en estos años son los siguientes

\begin{tabular}{cc}
\hline \multicolumn{2}{c}{ Bautismos (1527-1538) } \\
\hline Año & Bautismos \\
\hline 1527 & 54 \\
1528 & 76 \\
1529 & 35 \\
1530 & 55 \\
1531 & 57 \\
1532 & 63 \\
1533 & 57 \\
1534 & 95 \\
1535 & 65 \\
1536 & 75 \\
1537 & 82 \\
1538 & 106 \\
\hline
\end{tabular}

Es evidente la falta de regularidad en la serie, y como no disponemos de datos relativos a esta época en estudios sobre la población extremeña para saber si esas oscilaciones fueron generales en la región o, por el contrario, una circunstancia específica de Mérida, vamos a compararlos gráficamente con los que expone $\mathrm{Nadal}^{2}$ de una parroquia de Gerona.

La representación gráfica nos muestra cómo, a pesar de la diferencia cuantitativa entre ambas poblaciones, las dos sufren una paralela irregularidad, aunque la gran depresión que se manifiesta en Mérida en el año 1529, se adelante en un año a otra de semejante intensidad en Gerona. También hemos insertado las líneas de tendencia correspondiente a cada serie, siendo las dos crecientes, lo que coincide con la idea del incremento de la población española durante aquella centuria y, además, nos indica

2 Nadal, J., La pobiación española (siglos XVI a XX). Barcelona, 1973, pág. 21. 
que las oscilaciones de la misma eran bastante homogéneas dentro del territorio peninsular, al menos durante esta corta etapa.

\section{Bautismos en Mérida y Gerona}

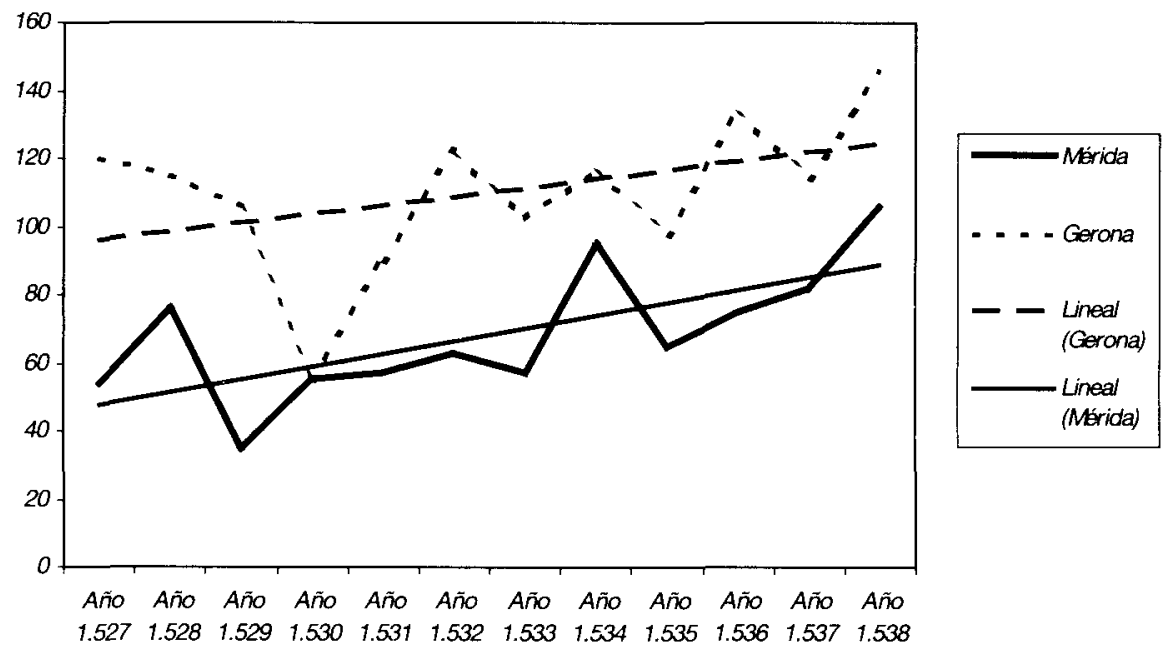

Si pasamos a tabular los bautismos de Mérida realizados entre Junio de 1526 hasta Julio de 1539, la muestra más amplia de que disponemos, y los agrupamos por meses, tanto en valores absolutos como porcentuales, tenemos la siguiente tabla:

\begin{tabular}{lr}
\hline \multicolumn{2}{l}{ Bautismos Mensuales (1526-1539) } \\
\hline Meses & \multicolumn{1}{c}{ Bautismos } \\
\hline - Enero & $105<>11^{\prime} 8 \%$ \\
- Febrero & $92<>10^{\prime} 3 \%$ \\
- Marzo & $50<5,6 \%$ \\
- Abril & $36<>4 ' 0 \%$ \\
- Mayo & $59<6^{\prime} 6 \%$ \\
- Junio & $51<>5 ' 7 \%$ \\
- Julio & $65<>7^{\prime} 3 \%$ \\
- Agosto & $81<>9^{\prime} 1 \%$ \\
- Septiembre & $83<>9^{\prime} 3 \%$ \\
- Octubre & $95<>10^{\prime} 6 \%$ \\
- Doviembre & $91<>10^{\prime} 2 \%$ \\
\hline
\end{tabular}


La irregularidad no es sólo una característica interanual, sino también intermensual. Para interpretar este proceso, al establecer una correlación directa entre bautismos y nacimientos, aun sabiendo que posiblemente era frecuente que muchos niños muriesen sin haber podido recibir el bautismo, aunque en algunas actas se hace mención que por la gravedad de su estado había sido bautizado en casa recién nacido, vemos que éstos presentan dos ciclos diferentes dentro del año: uno, de Agosto a Febrero, con muchos nacimientos; y otro, de Marzo a Julio, con pocos. Al retroceder los nacimientos a los meses en que se produjeron las correspondientes concepciones, para lo cual hemos establecido, en primer lugar, en 15 días la diferencia entre nacimiento y bautismo, y, en segundo lugar, 270 días entre la concepción y el nacimiento, obtenemos la tabla siguiente:

\begin{tabular}{cccc}
\hline \multicolumn{4}{c}{ Concepciones Mensuales (1525-1538) } \\
\hline Mes & $N^{\circ}$ concepciones & Media mensual & Porcentaje \\
\hline Enero & 85 & $6^{\prime} 5$ & $9^{\prime} 6 \%$ \\
Febrero & 86 & $6^{\prime} 6$ & $9^{\prime} 7 \%$ \\
Marzo & 79 & $6^{\prime} 1$ & $8^{\prime} 9 \%$ \\
Abril & 102 & $7^{\prime} 8$ & $1^{\prime} 6 \%$ \\
Mayo & 91 & $7^{\prime} 0$ & $10^{\prime} 3 \%$ \\
Junio & 39 & $3^{\prime} 0$ & $4^{\prime} 4 \%$ \\
Julio & 49 & $3^{\prime} 8$ & $5^{\prime} 5 \%$ \\
Agosto & 51 & $3^{\prime} 6$ & $5^{\prime} 8 \%$ \\
Septiembre & 54 & $4^{\prime} 3$ & $6^{\prime} 1 \%$ \\
Octubre & 66 & $5^{\prime} 2$ & $7^{\prime} 5 \%$ \\
Noviembre & 89 & $6^{\prime} 8$ & $10^{\prime} 1 \%$ \\
Diciembre & 92 & $7^{\prime} 0$ & $10^{\prime} 4 \%$ \\
\hline
\end{tabular}

Como es lógico, también aquí vemos dos períodos diferentes, uno alto, de Noviembre a Mayo, y otro bajo, de Junio a Octubre. Esto, en una primera aproximación, nos da testimonio de una sociedad que vivía en relación muy directa con las horas de sol de cada día y, también, con las necesidades laborales de las tareas agrícolas; pero avanzando en el análisis de los datos, y al ver cómo el mes de Febrero, un $10 \%$ menor en días que los de Enero y Marzo, tiene, sin embargo, una media mayor que éstos, nos lleva a pensar que en la producción de las concepciones, aparte de! clima y los trabajos agrícolas, también había una notoria influencia de las mentalidades, que en estos meses de Febrero a Mayo, $y$ variando de un año a otro, tenían que hacer frente a dos acontecimientos contrapuestos entre sí: por un lado, y primero en el tiempo, las 
fiestas de Carnestolendas, e inmediatamente el otro, más largo, constituido por la Cuaresma y la Semana Santa; mientras el Carnaval acentuaría las relaciones carnales y, en consecuencia, aumentarian las concepciones, y como estos festejos se producen siempre en Febrero, tenemos ya una explicación relativa a la abundancia evidenciada en este mes; la abstinencia en la Cuaresma reduciría considerablemente las concepciones, produciéndose otro incremento al finalizar la Semana Santa e iniciarse las fiestas de Pascua, como indican los valores medios de Abril y Mayo.

Concepciones mensuales (1.525-1.538)

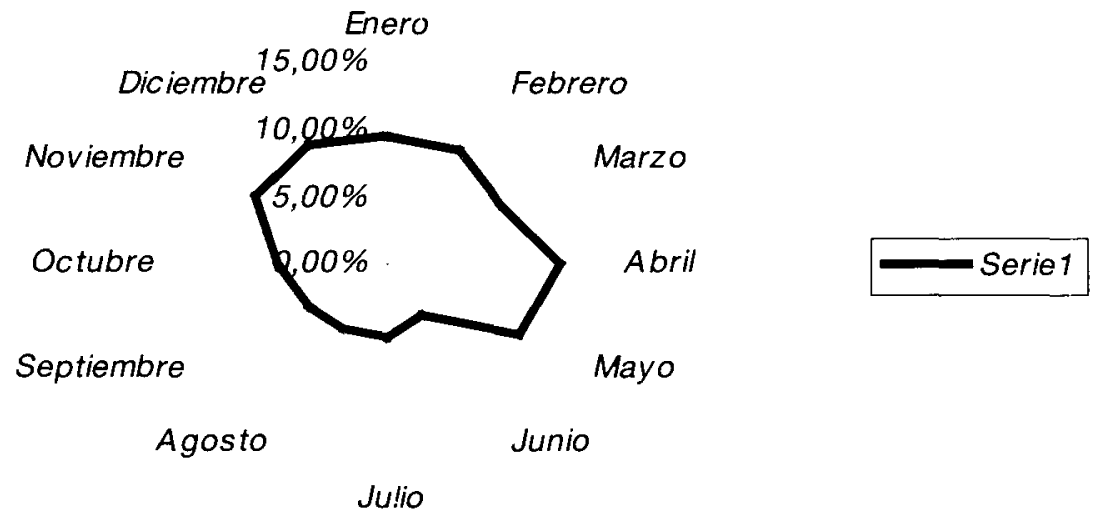

La distribución de nacimientos por sexos es ligeramente favorable al femenino, lo que medido por el parámetro demográfico de la Razón de Masculinidad nos da como valor 0'98.

Los registros bautismales no nos pueden informar de las familias que no tienen hijos, $y$, por otra parte, al ser tan reducido el período que comprenden los mismos, es lógico que de las familias que aparecen, unas ya hubieran iniciado su ciclo de fertilidad antes de 1526 y otras lo finalizaran después de 1539; es decir, de la relación de hijos y familias que se identifican no se puede extraer una conclusión precisa acerca del número de hijos que las componian, además la movilidad de los vecinos al cambiar de domicilio dentro de la localidad hacía que, en este caso, al irse a la Parroquia de Santa Olalla, registrasen allí los nuevos nacimientos, así como otros que ya tenían hijos bautizados en ésta parroquia, se mudaban a la de Santa María y tenían otros bautizados en ésta: pero como una aproximación a esta importante cuestión, a la que si podremos aportar una res- 
puesta con los datos de la segunda mitad de la centuria, mostraremos los siguientes valores:

\begin{tabular}{lcc}
\hline Composición familiar & $\mathbf{N}^{\circ}{ }^{\circ}$ de familias & N. ${ }^{\circ}$ de hijos \\
\hline - Familias con 1 hijo & 409 & 409 \\
- Familias con 2 hijos & 105 & 210 \\
- Familias con 3 hijos & 60 & 180 \\
- Familias con 4 hijos & 13 & 52 \\
- Familias con 5 hijos & 8 & 40 \\
- Familias con 6 hijos & 1 & 6 \\
\hline Totales & 596 & 897 \\
\hline
\end{tabular}

De estos datos obtenemos una razón de 1'5 hijos por familia fértil, puesto que no conocemos las familias estériles, que sumados al padre y la madre nos daría un conjunto de habitantes por hogar de 3'5, cifra realmente baja y que nos afirma en la idea de que los resultados que hemos obtenido de la composición familiar son defectuosos, en cuanto faltan hijos en esas familias, nacidos antes o después de las fechas de inicio y final de estos registros bautismales estudiados. Estos valores realmente bajos, al conocer nominalmente a las familias y ver la secuencia de los nacimientos, nos ponen en presencia de la mortalidad infantil, puesto que en una misma familia es muy frecuente el hecho de que se repita el nombre de uno o varios hijos, lo que nos hace pensar que los que aparecen repetidos sustituyen a otro hermano nacido anteriormente y bautizado con ese nombre. También se intuye la muerte de hombres y mujeres, y la producción de nuevos matrimonios (viudo-viuda, soltero-viuda, viudo-soltera) que, en cualquier caso, no suponen la formación de una nueva familia, por lo que, de haber existido registros matrimoniales, se habrían confirmado estas uniones y habríamos podido reducir el número de famiiias, aumentando correlativamente la razón Hijos/Familia.

Por último, en lo que respecta a esta primera mitad del siglo xVI, señalaremos que hemos localizado 18 casos de nacimientos de gemelos o mellizos, y este dato nos permite distinguir entre partos sencillos y partos múltiples, aunque sólo hemos detectado los dobles, y tampoco descartamos que éstos sean más numerosos, pues también era posible que en alguno de ellos falleciera alguno de los niños, y en este caso el bautismo del superviviente aparece como resultado de un parto sencillo. 


\section{Partos (I)}

\begin{tabular}{ccc}
\hline Tipo de parto & N. ${ }^{\circ}$ de partos & Porcentaje \\
\hline - Partos sencillos & 874 & $98,98 \%$ \\
- Partos dobles & 9 & $1{ }^{\prime} 02 \%$ \\
\hline Totales & 883 & 100 \\
\hline
\end{tabular}

\section{SEGUNDA MITAD DEL SIGLO XVI}

Para la segunda mitad del siglo xvi contamos, como se ha indicado, con registros bautismales desde el año 1558 hasta 1600 en la Parroquia de Santa María, aunque faltan los del año 1560 (completo) y parte de los años 1575, 1576 y 1577; en la Parroquia de Santa Olalla los registros comienzan en el año 1581. Tanto en una como en otra, dentro de los libros de bautismo aparecen actas de confirmaciones conjuntas para ambas parroquias, las cuales también proporcionan una información aprovechable para identificar, contrastar y completar composiciones familiares.

El resumen numérico de los registros bautismales, con la desagregación de los adultos que han recibido el sacramento bautismal, nos da la cifra de los niños nacidos, y es el siguiente:

\begin{tabular}{lr}
\hline \multicolumn{2}{c}{ Bautismos y Nacimientos (1558-1600) } \\
\hline - Registros bautismales & 5767 \\
- Adultos bautizados & 24 \\
\hline Nacimientos correspondientes & 5743 \\
\hline
\end{tabular}

La distribución por sexos de los nacimientos

\begin{tabular}{lr}
\hline - Niños & 2904 \\
- Niñas & 2832 \\
- Sin identificar & 7 \\
\hline RAZÓN DE MASCULINIDAD & 1 '02 \\
\hline
\end{tabular}


De las actas de Confirmación incluidas en los libros de bautismos, no solamente hemos recogido información para completar las fichas familiares, sino que también hemos tomado datos para calcular la relación entre sexos de la población infantil, y con ellos hemos confeccionado la siguiente tabla:

\begin{tabular}{cccc}
\hline \multicolumn{4}{c}{ Confirmaciones } \\
\hline \multirow{2}{*}{ AÑO } & $\begin{array}{c}\text { NIÑOS } \\
\text { CONFIRMADOS }\end{array}$ & $\begin{array}{c}\text { NIÑAS } \\
\text { CONFIRMADAS }\end{array}$ & $\begin{array}{c}\text { RAZÓN DE } \\
\text { MASCULINIDAD }\end{array}$ \\
\hline \multirow{2}{*}{1564} & 228 & 245 & 0 \\
1582 & 118 & 94 & 1 '25 \\
1583 & 4 & 4 & 1 '00 \\
1587 & 104 & 90 & 1,16 \\
\hline Totales & 454 & 433 & 1 '05 \\
\hline
\end{tabular}

Como vemos en este dato final, media de todas las confirmaciones realizadas, al comparar con el que habíamos obtenido en el cómputo general de los bautizados, que era de 1.02 , el que proporcionan las confirmaciones es superior, y esto se puede interpretar, al establecer una correlación entre la población bautizada y la confirmada, estimando que se trata de la misma pero con una diferencia de pocos años entre una ceremonia y otra, que ha disminuido ligeramente la población femenina (más atacada por la mortalidad) que la masculina, al variar positivamente la Razón de Masculinidad de $1^{\prime} 02$ a 1'05, lo que supone un incremento del 2'9\%.

El número de mellizos o gemelos registrados, ha sido de 66 , lo que representa el $1^{\prime} 1 \%$ del total de los nacimientos, y que respecto a los partos nos da el siguiente cuadro:

\begin{tabular}{ccc}
\hline & Partos $(\mathbf{I})$ & \\
\hline Tipo de parto & N. ${ }^{\circ}$ de partos & Porcentaje \\
\hline $\begin{array}{c}\text { - Partos sericillos } \\
\text { Partos dobles }\end{array}$ & $\begin{array}{r}5.680 \\
33\end{array}$ & $\begin{array}{r}94 \\
0^{\prime}\end{array}$ \\
\hline Totales & 5.713 & 100 \\
\hline
\end{tabular}

Otros datos de interés para el estudio de la población, son los siguientes: 


\begin{tabular}{lrl}
\hline - Hijos de padre desconocido & 350 & $6^{\prime} 1 \%$ \\
- Hijos de madre desconocida & 26 & $0^{\prime} 4 \%$ \\
- Expósitos & 47 & $0^{\prime} 8 \%$ \\
- Moriscos & 290 & $5^{\prime} 0 \%$ \\
\hline
\end{tabular}

Los bautismos anuales realizados durante esta etapa los exponemos en la tabla y acompañamos del gráfico correspondiente:

\begin{tabular}{cccc}
\hline \multicolumn{4}{c}{ Bautismos 1561-1600 } \\
\hline AÑO & BAUTISMOS & AÑO & BAUTISMOS \\
\hline 1561 & 99 & 1581 & 125 \\
1562 & 141 & 1582 & 170 \\
1563 & 144 & 1583 & 152 \\
1564 & 128 & 1584 & 175 \\
1565 & 102 & 1585 & 203 \\
1566 & 131 & 1586 & 163 \\
1567 & 147 & 1587 & 196 \\
1568 & 103 & 1588 & 188 \\
1569 & 164 & 1589 & 188 \\
1570 & 147 & 1590 & 131 \\
1571 & 109 & 1591 & 149 \\
1572 & 175 & 1592 & 146 \\
1573 & 113 & 1593 & 179 \\
1574 & 147 & 1594 & 164 \\
1575 & 58 & 1595 & 191 \\
1576 & 59 & 1596 & 162 \\
1577 & 33 & 1597 & 180 \\
1578 & 77 & 1598 & 178 \\
1579 & 89 & 1599 & 169 \\
1580 & 103 & 1600 & 170 \\
\hline
\end{tabular}
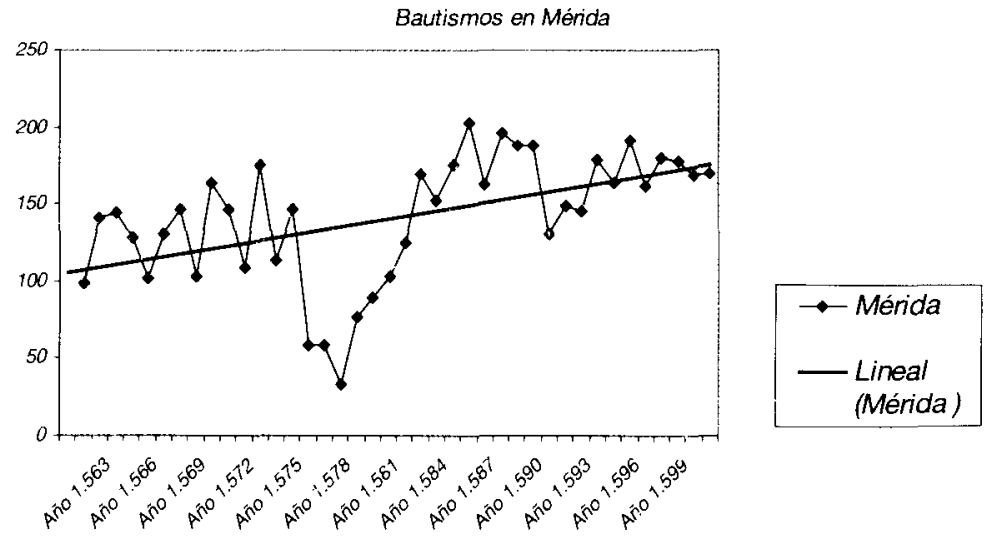
La línea de tendencia insertada manifiesta una pendiente positiva, testimonio del crecimiento de la población, aunque hay una depresión acusada en los años 1575, 1576 y 1577, de los que ya hemos dicho que faltan algunos registros. Como en la primera mitad del siglo, son constantes los dientes de sierra representativos de la irregularidad.

De esta segunda mitad de la centuria conocemos monografías y artículos, que ya dan una precisa información de los bautismos en otras localidades extremeñas, como en Badajoz, Almendralejo o un conjunto de 37 pueblos entre los que no se encuentran Badajoz, Almendralejo, ni Mérida ${ }^{3}$; para efectuar las comparaciones tomaremos el período comprendido entre 1581 y 1600 , porque es del que poseemos datos representativos de la totalidad de la población emeritense al sumar los de las dos parroquias, y para una mejor estimación de las diferencias porcentuales entre las tres series de datos, hemos elaborado una tabla de números índice, tomando como base el año 1581, que también representamos gráficamente:

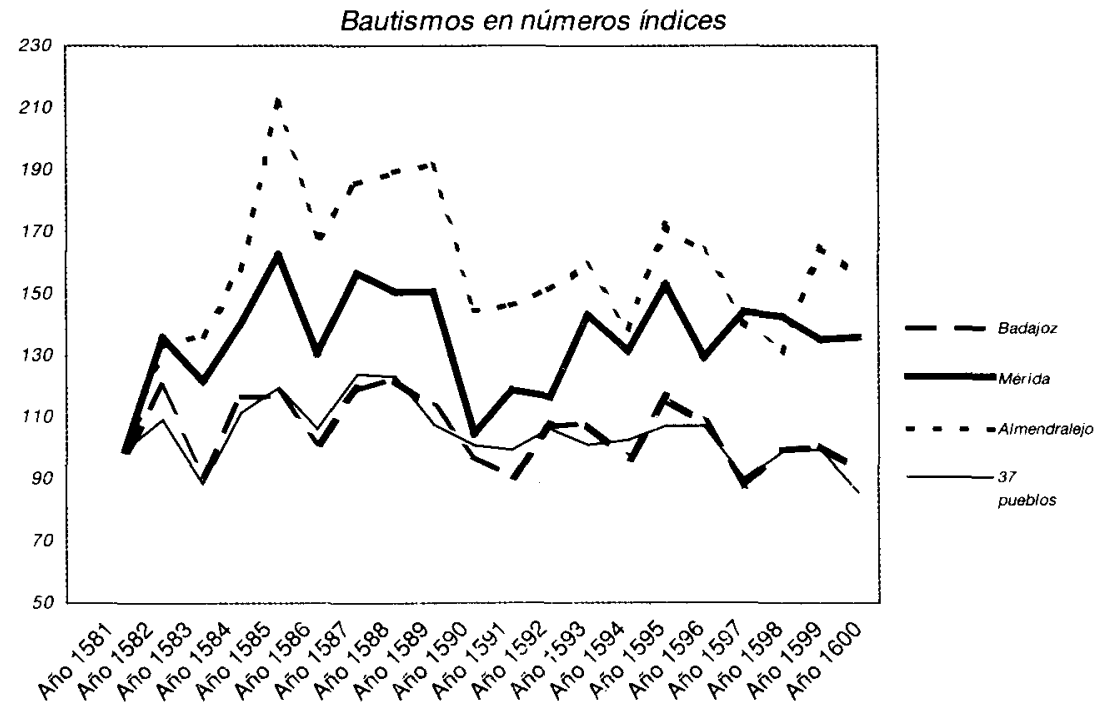

3 CoRtés Cortés, F, Una ciudad de frontera. Badajoz en los siglos xVI y xvII. Badajoz, 1990, págs. 64 y 65 .

Zarandieta Arenas, F., Aimendralejo en los siglo xvi y xvil. Almendralejo, 1993, págs. 138 a
143.
Llopis, E., Melón, M. A., Rodriguez, M., Rodriguez, A. y Zarandieta, F., «El movimiento de la
población extremeña durante el Antiguo Régimen», Revista de Estudios Extremeños, Tomo XLIX,
Num. 1, Badajoz, 1992, págs. 206 y 207 . 


\begin{tabular}{|c|c|c|c|c|c|c|c|c|}
\hline \multicolumn{9}{|c|}{ Bautismos en Extremadura } \\
\hline \multirow{2}{*}{ AÑO } & \multicolumn{2}{|c|}{ MÉRIDA } & \multicolumn{2}{|c|}{ BADAJOZ } & \multicolumn{2}{|c|}{ ALMENDRALEJO } & \multicolumn{2}{|c|}{37 PUEBLOS } \\
\hline & Bautismo & Índice & Bautismos & indice & Bautismos & Índice & Bautismos & İndice \\
\hline 1581 & 125 & 100 & 365 & 100 & 82 & 100 & 2416 & 100 \\
\hline 1582 & 170 & 136 & 438 & 120 & 109 & 133 & 2646 & 110 \\
\hline 1583 & 152 & 122 & 332 & 91 & 112 & 137 & 2133 & 88 \\
\hline 1584 & 175 & 140 & 425 & 116 & 129 & 157 & 2690 & 111 \\
\hline 1585 & 203 & 162 & 427 & 117 & 174 & 212 & 2888 & 120 \\
\hline 1586 & 163 & 130 & 371 & 102 & 138 & 168 & 2563 & 106 \\
\hline 1587 & 196 & 157 & 434 & 119 & 152 & 185 & 3004 & 124 \\
\hline 1588 & 188 & 150 & 448 & 123 & 155 & 189 & 2971 & 123 \\
\hline 1589 & 188 & 150 & 411 & 113 & 157 & 191 & 2612 & 108 \\
\hline 1590 & 131 & 105 & 355 & 97 & 119 & 145 & 2434 & 101 \\
\hline 1591 & 149 & 119 & 330 & 90 & 120 & 146 & 2406 & 100 \\
\hline 1592 & 146 & 117 & 390 & 107 & 124 & 151 & 2573 & 106 \\
\hline 1593 & 179 & 143 & 394 & 108 & 130 & 159 & 2451 & 101 \\
\hline 1594 & 164 & 131 & 353 & 97 & 113 & 138 & 2473 & 102 \\
\hline 1595 & 191 & 153 & 423 & 116 & 141 & 172 & 2588 & 107 \\
\hline 1596 & 162 & 130 & 393 & 108 & 134 & 163 & 2584 & 107 \\
\hline 1597 & 180 & 144 & 321 & 88 & 116 & 141 & 2181 & 90 \\
\hline 1598 & 178 & 142 & 364 & 100 & 107 & 130 & 2383 & 99 \\
\hline 1599 & 169 & 135 & 368 & 101 & 135 & 165 & 2403 & 99 \\
\hline 1600 & 170 & 136 & 338 & 93 & 129 & 157 & 2071 & 86 \\
\hline
\end{tabular}

Este gráfico nos muestra dos grupos de poblaciones, el formado por Badajoz y las 37 localidades, que manifiestan una gran estabilidad, sin grandes altibajos y con una tendencia ligeramente decreciente; y el otro, constituido por Almendralejo y Mérida, con altibajos más acusados y ambas con tendencia creciente. Este despegue de estas dos poblaciones con respecto al otro amplio conjunto pudo deberse, entre otras cosas, a la llegada de moriscos de los repartidos de Granada tras la guerra de las Alpujarras, de los que no se hace ninguna mención ni en Badajoz ni en el artículo de los 37 pueblos.

Vista la evolución de los bautismos en distintas localidades de Extremadura, tiene interés conocer la del conjunto, es decir, Mérida, Almendralejo, Badajoz y las 37 localidades, que son Aceuchal, Azuaga, Bodonal de la Sierra, Cabeza del Buey, Calera de León, Campanario, Fuente de Cantos, Fuente del Maestre, Jerez de los Caballeros, Montemolín, Monterrubio de la Serena, Puebla del Prior, Solana de los Barros, Talavera la Real, Torre de Miguel Sesmero, Valverde de Mérida, Villagarcía de la Torre, Zafra, Alcuéscar, Aliseda, Almoharín, Arroyomolinos de Montánchez, Brozas, Calzadilla, Casar de Cáceres, Guadalupe, Guijo de Galisteo, Losar de la Vera, Malpartida de Cáceres, Mata de Alcantara, Membrío, Monroy, Navaconcejo, Torrejoncillo, 
Villanueva de la Sierra, Villanueva de la Vera y Villar del Pedroso ${ }^{4}$, conjunto que comprende 43 parroquias, y cuyos valores tabulamos seguidamente:

\begin{tabular}{cccc}
\hline \multicolumn{4}{c}{ Bautismos en Extremadura (1581-1600) } \\
\hline AÑO & N. $^{\circ}$ BAUTISMOS & AÑO & N. $^{\circ}$ BAUTISMOS \\
\hline 1581 & 2988 & 1591 & 3005 \\
1582 & 3363 & 1592 & 3233 \\
1583 & 2729 & 1593 & 3154 \\
1584 & 3419 & 1594 & 3103 \\
1585 & 3692 & 1595 & 3341 \\
1586 & 3235 & 1596 & 3273 \\
1587 & 3786 & 1597 & 2798 \\
1588 & 3762 & 1598 & 3032 \\
1589 & 3368 & 1599 & 3075 \\
1590 & 3039 & 1600 & 2708 \\
& & & 3072 \\
\hline
\end{tabular}

Y la representación gráfica de la serie extremeña y sus medias decenales, nos muestra que el conjunto de los nacimientos disminuyó en la úl. tima década de la centuria, por lo que se puede deducir, de otra parte, que la dinámica de nacimientos de Mérioia y Aimendralejo era distinta de la del conjunto extremeño

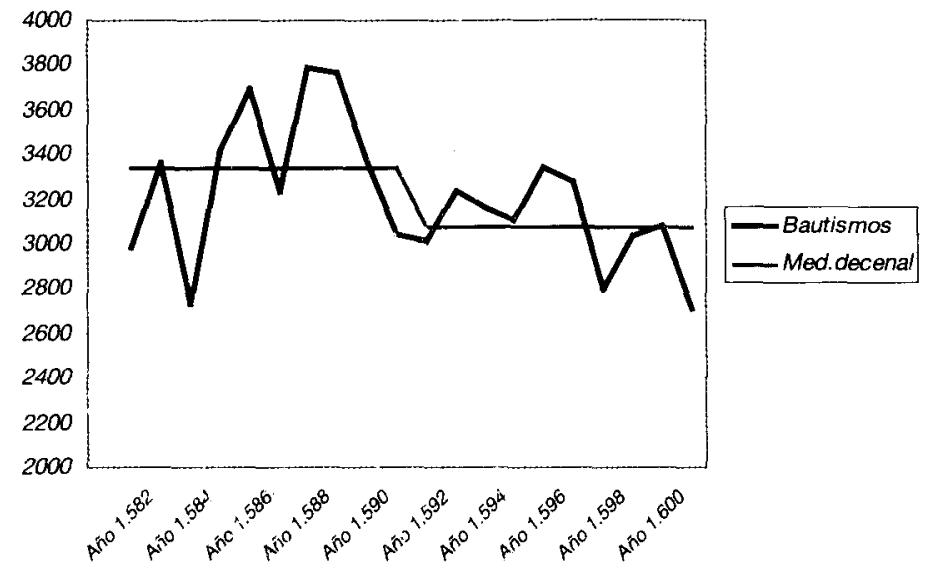

4 LloPIs y OTROs., Ob. cit., págs. 190 y 191. 
Nadal, en un estudio donde propone un balance regional de la población española ${ }^{5}$, toma datos para la región extremeña de 8 archivos parroquiales (Badajoz, Campanario, Jerez de los Caballeros, Logrosán, Navalmoral de la Mata y Villar del Pedroso), muestra realmente pequeña frente a la que nosotros estamos tratando; por ello vamos a comparar las dos, y esto hará necesario previamente establecer una base común para los índices ${ }^{6}$, pues Nadal no da los valores absolutos, sino números índice, lo que requiere transformar la nuestra, también, en una serie de números índices y además, cambiar a la de Nadal la base, pues la tiene establecida en la media decenal de 1620-1629, década que está fuera del período que comparamos, por lo que la tabla de comparación tiene la base 100 en la media decenal de 1581-1590, y esto permite la comparación de ambas series.

\begin{tabular}{|c|c|c|c|c|c|}
\hline \multicolumn{6}{|c|}{ Bautizados en Extremadura } \\
\hline AÑo & Índice Propio & Índice Nadal & AÑo & Índice Propio & Índice Nadal \\
\hline 1581 & $88^{\prime} 63$ & $91 ' 61$ & 1591 & $89 ' 13$ & $100^{\prime} 64$ \\
\hline 1582 & $99^{\prime} 75$ & $83 \cdot 95$ & 1592 & 9590 & $110^{\prime} 44$ \\
\hline 1583 & $80 ' 95$ & $82 ' 66$ & 1593 & $93 ’ 55$ & $88^{\prime} 57$ \\
\hline 1584 & $101^{\prime} 41$ & $99 ' 45$ & 1594 & $92 ' 04$ & $101 ' 13$ \\
\hline 1585 & $109 ' 51$ & $102^{\prime} 26$ & 1595 & $99^{\prime} 10$ & $93^{\prime} 47$ \\
\hline 1586 & $95 ' 86$ & $92 ' 55$ & 1596 & $97 ' 08$ & $97^{\prime} 66$ \\
\hline 1587 & $112^{\prime} 30$ & $96 ' 44$ & 1597 & $82 ' 99$ & $73 ' 59$ \\
\hline 1588 & $111^{\prime} 59$ & $125 \cdot 33$ & 1598 & $899^{\prime} 94$ & $83 ' 51$ \\
\hline 1589 & $99^{\prime} 90$ & $130^{\prime} 58$ & 1599 & $91 ' 21$ & $92 ' 45$ \\
\hline 1590 & $90 ' 14$ & $95^{\prime} 22$ & 1600 & $80 ' 33$ & $766^{\prime} 91$ \\
\hline
\end{tabular}

Es evidente que hay bastante correlación entre ambas series, con una primera década creciente seguida de otra de depresión, aunque la dispersión en la serie de Nadal es mayor, lo cual es lógico al ser la muestra mucho menor que la utilizada por nosotros, por lo que estimamos que la conjunta de 43 parroquias es la mas representativa de la población regional de Extremadura.

Cuya representación gráfica es la siguiente:

5 NADAL, J., "La población española durante los siglos XVI, XVII y XVIII. Un balance a escala regional», en PÉREZ. MOREDA, V. y REHER, D-S., Demografía histórica española. Madrid, 1988, págs. 39 a 54 .

- Ballesteros Diez, J. A., "Métodos cuantitativos en las Ciencias Sociales (1)". Proserpina, N. ${ }^{\circ}$ 13. UNED, 1996, págs. 31 a 45. 


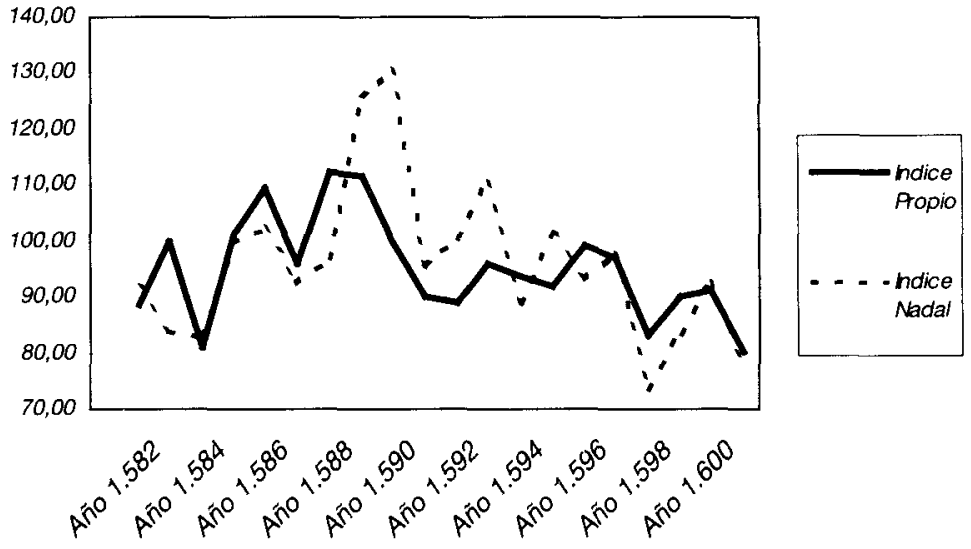

\section{LA NATALIDAD}

Para afinar en el estudio demográfico, al hacer la traslación de la fecha del bautismo a la del nacimiento, hemos restado a aquella 15 días, pues igual que en la primera parte del siglo tampocc en esta segunda mitad se detalla en la inscripción la del nacimiento, y al tener referencias de que ese intervalo en unas diócesis era de ocho días y en otras llegaba a tres semanas, hemos elegido la quincena como valor intermedio; conseguida de este modo la fecha del nacimiento, le restamos 270 días y encontramos la fecha de la concepción.

En primer lugar vamos a comparar la correlación existente entre la distribución de nacimientos por meses que determinamos para la primera mitad del siglo y la que se produce en la segunda:

\begin{tabular}{|c|c|c|}
\hline \multicolumn{3}{|c|}{ Nacimientos mensuales (en porcentaje) } \\
\hline Mes & Nacimientos $1527-1538$ & Nacimientos $1558-1600$ \\
\hline - Enero & $11 ' 8 \%$ & $10^{\prime} 9 \%$ \\
\hline - Febrero & $103 \%$ & $9^{\prime} 2 \%$ \\
\hline - Marzo & $5 ' 6 \%$ & $7 ' 8 \%$ \\
\hline - Abril & $40 \%$ & $6^{\prime} 9 \%$ \\
\hline - Mayo & $6^{\prime} 6 \%$ & $5 ' 8 \%$ \\
\hline - Junio & $5 \cdot 7 \%$ & $5 \cdot 4 \%$ \\
\hline - Julio & $7 ’ 3 \%$ & $7 ' 2 \%$ \\
\hline - Agosto & $9 \cdot 1 \%$ & $9 ' 2 \%$ \\
\hline - Septiembre & $9 ' 3 \%$ & $9 \cdot 5 \%$ \\
\hline - Octubre & $10^{\prime} 6 \%$ & $9 ; \%$ \\
\hline - Noviembre & $102 \%$ & $87 \%$ \\
\hline - Diciembre & $9 ' 4 \%$ & $9 ' 8 \%$ \\
\hline
\end{tabular}


Cuya visualización gráfica exponemos seguidamente:

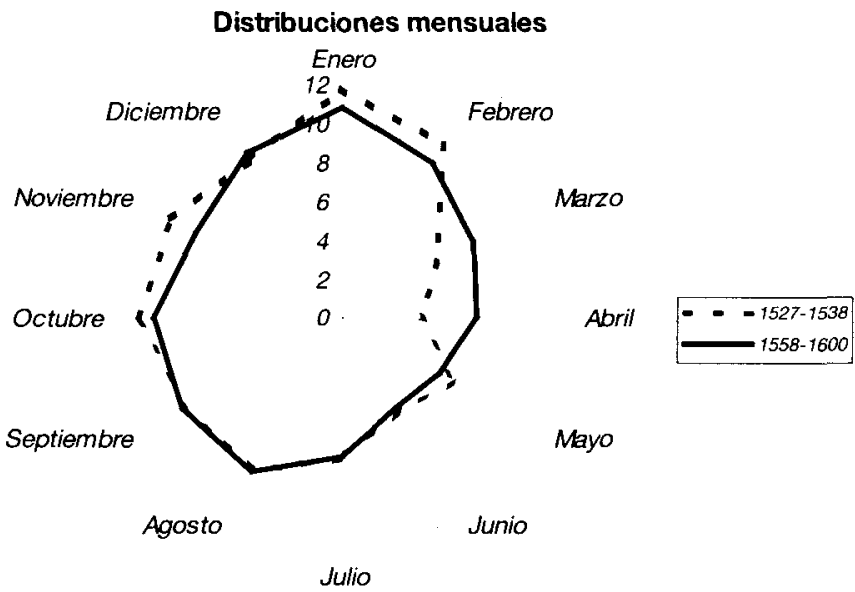

evidenciándose que la distribución mensual de los nacimientos entre 1558 y 1600 es más regular que entre 1527 y 1538; medidas las dispersiones de cada serie, tenemos como valores de la desviación, 1'6 en correspondiente a la segunda mitad del siglo y 2'3 en la de la primera mitad; sigue siendo Enero el mes donde se producen más nacimientos, pero frente al $11 ' 8 \%$ primero hay, ahora, 10 ' $9 \%$; y si el mínimo se daba en el mes de Abril con el $40 \%$, en la segunda mitad del siglo se producirá en Mayo, pero con el 5 ' $4 \%$ de los nacimientos.

Antes de cerrar este epígrafe dedicado a la natalidad, señalaremos los nacimientos generalmente conocidos como ilegítimos, aunque esta calificación sea muy discutible, pero no es este el espacio para ese debate y seguiremos la costumbre establecida. Los datos de nacimientos producidos en esta circunstancias son los siguientes:

\begin{tabular}{ccc}
\hline \multicolumn{3}{c}{ Natalidad ilegitima } \\
\hline Filiación & $\mathbf{1 5 2 7 - 1 5 3 8}$ & $\mathbf{1 5 5 5 - 1 6 0 0}$ \\
\hline - Hijos de padre desconocido & $7<0^{\prime} 8 \%$ & $350<>6^{\prime} 1 \%$ \\
- Hijos de madre desconocida & $28<>3^{\prime} 1 \%$ & $26<>0^{\prime} 4 \%$ \\
- Expósitos & 0 & $47<>0^{\prime} 8 \%$ \\
\hline Total ilegitimidad & $35<>3{ }^{\prime} 9 \%$ & $423<>77^{\prime} 3 \%$ \\
\hline
\end{tabular}


Datos estos que nos muestran un cambio importante en las costumbres sociales, por cuanto casi se ha duplicado la natalidad extramatrimonial, y, lo que es más llamativo, si en la primera mitad de la centuria no habían aparecido expósitos, en la segunda son ya el 0'8\% del total de los nacidos. Todos estos datos dan pie a estudios de historia social que no son el objeto de este artículo, pero que tienen mucho interés.

Otro gran grupo es el de los moriscos, que aunque había algunos presentes viviendo de modo estable en Mérida en el primer tercio del siglo, aparecen cuantiosamente en el último tercio, y en total hemos contado 290 nacimientos, lo que supone el $5 \%$ del total.

\section{LA NUPCIALIDAD}

Los libros de ambas parroquias cubren muy pocos años dentro del siglo XVI, pues los registros en Santa María se inician en el año 1594 y los de Santa Olalla en 1595, por lo que los datos que aportan no podemos interpretarlos en rigor como representativos de la centuria, pero indudablemente nos darán unas indicaciones acerca de la población que contraía matrimonio.

En primer lugar, en relación con el estado de los contrayentes, hemos determinado los siguientes porcentajes:

\begin{tabular}{lc}
\hline \multicolumn{2}{c}{ Contrayentes } \\
\hline Estados prenupciales & Porcentaje \\
\hline Soltero con soltera & $83^{\prime} 8 \%$ \\
Viudo con soltera & $7^{\prime} 4 \%$ \\
Viudo con viuda & $7^{\prime} 4 \%$ \\
Soltero con viuda & $1^{\prime} 4 \%$ \\
\hline
\end{tabular}

Es decir, la situación de viudedad de uno, o de los dos contrayentes, se presentaba en el 16 ' $2 \%$ de los enlaces matrimoniales.

Respecto a las edades de los contrayentes, determinadas al cruzar las tablas de datos bautismales con las de matrimonio, porque en el registro de estos no se hacía constar ese dato, hemos calculado los siguientes datos, entendidos en relación al primer matrimonio:

- Homibres: a ios 27'1 años

- Mujeres: a los 22'7 años 


\section{RELACION ESTADOS PRENUPCIALES}

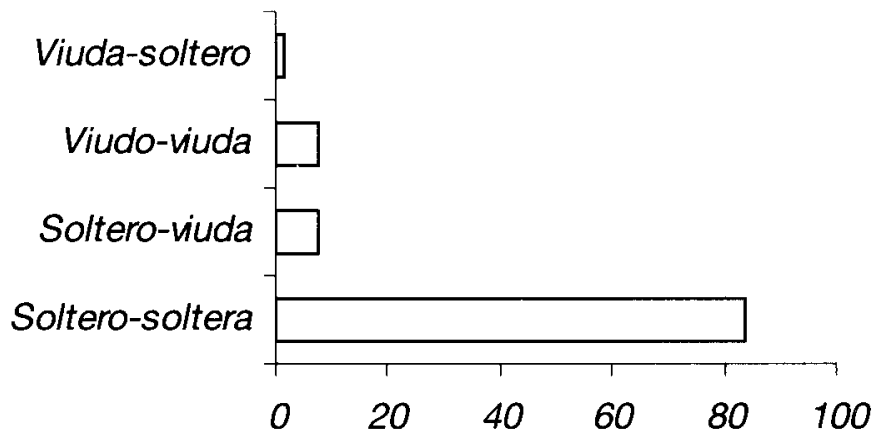

Cómo los datos que hemos manejado para las uniones matrimoniales comienzan en una parroquia en 1594 y en la otra en 1595, no podemos dar las edades calculadas para el acceso al matrimonio como representativas del siglo $X V I$, pero para estimar si había semejanza en algún lugar próximo, hemos visto que en Almendralejo, en la década de 1611 a 1620 , la media de edad de los varones era de $27^{\prime} 1$ años, y la de las mujeres $22^{\prime} 9^{7}$, como vemos prácticamente iguales a las de Mérida.

Al analizar estadísticamente la serie de datos correspondiente a los varones, hemos visto que la dispersión es de 5'27, frente a la de las mujeres que es de 6'0, o sea, el rango que cubren las mujeres al acceder al matrimonio es mayor que el de los hombres, y para avanzar más en éste análisis haremos una distribución de frecuencias en intervalos quinquenales

\begin{tabular}{|c|c|c|}
\hline \multicolumn{3}{|c|}{ Edades Nupciales } \\
\hline Rango del intervalo (años) & Hombres (\%) & Mujeres (\%) \\
\hline - 11 a 15 años & 0 & $5 \cdot 2 \%$ \\
\hline - 16 a 20 años & $121 \%$ & $36,2 \%$ \\
\hline - 21 a 25 años & $30,3 \%$ & $25 \cdot 9 \%$ \\
\hline - 26 a 30 años & $242 \%$ & $224 \%$ \\
\hline - 31 a 35 años & $24 ' 2 \%$ & $8^{\prime} 6 \%$ \\
\hline - 36 a 40 años & $9^{\prime} 1 \%$ & $17 \%$ \\
\hline
\end{tabular}

7 Zarandieta Arenas, F., Almenóralejo en los siglo xvi y xvil. Tomo I. Almendralejo, 1993, pág. 251. 
La visualización gráfica de esta distribución de frecuencias, nos evidencia cómo las mujeres acceden mucho antes al matrimonio que los varones; las dos más jovenes se casaron con una edad de 12'83 años y de 14 '33 años, mientras que las mujeres con más edad al llegar a la nupcialidad lo hicieron con 35'18, 34'80 y 34'37 años. En el grupo de los hombres, las edades más bajas son las de 17 '55 y 18'76 años, y las más altas fueron de $36^{\prime} 42$ y 35'67 años, y como vemos, el porcentaje de varones casados con edades comprendidas entre 31 y 40 años es muy superior al de las mujeres.

\section{Edades nupciales}

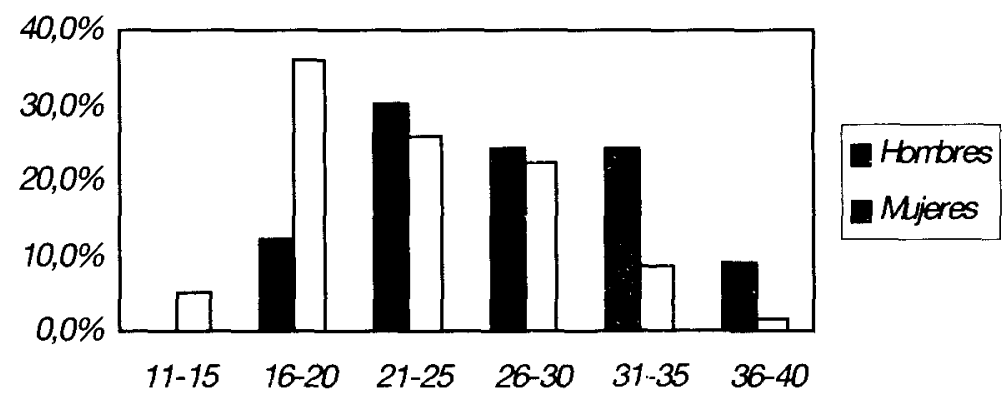

La distribución de la nupcialidad a lo largo de los meses del año nos da los siguientes porcentajes:

\begin{tabular}{|c|c|}
\hline \multicolumn{2}{|c|}{ Distribución mensual de la nupcialidad } \\
\hline Mes & Matrimonios (\%) \\
\hline - Enero & $10 ' 9 \%$ \\
\hline - Febrero & $12 \cdot 1 \%$ \\
\hline - Marzo & $84 \%$ \\
\hline - Abril & $8 ' 3 \%$ \\
\hline - Mayo & $8 ' 8 \%$ \\
\hline - Junio & $6 \cdot 4 \%$ \\
\hline - Julio & $5 \cdot 9 \%$ \\
\hline - Agosto & $5 ' 8 \%$ \\
\hline - Septiembre & $7^{\prime} 8 \%$ \\
\hline - Octubre & $8 ' 9 \%$ \\
\hline - Noviembre & $6 \cdot 6 \%$ \\
\hline - Diciembre & $10.1 \%$ \\
\hline
\end{tabular}


Y el gráfico de la misma es el siguiente:

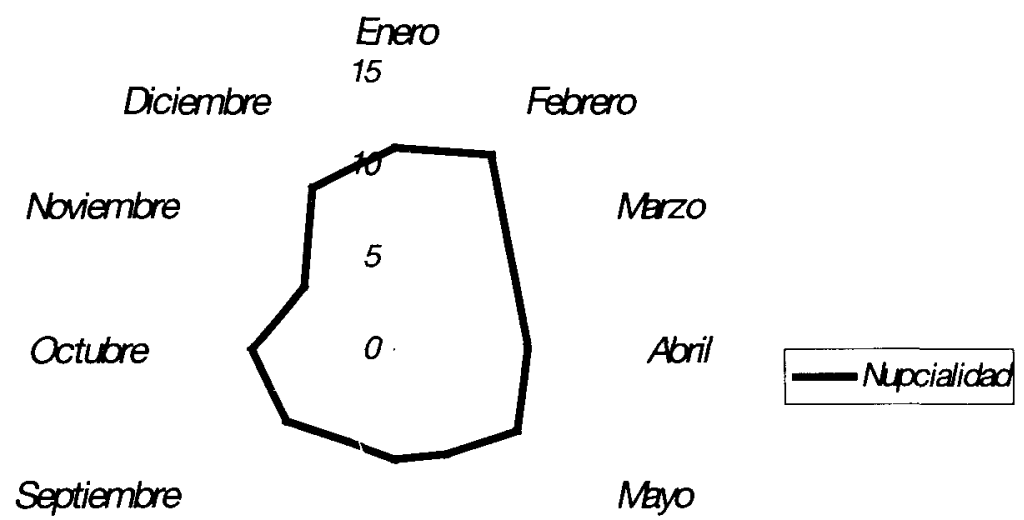

Agosto Junio

A la vista dei mismo se manifiesta una gran semejanza entre la distribución de los matrimonios, el 30'1\% se celebran de Diciembre a Febrero, con la de los nacimientos, pero al ser la serie de aquéllos mucho más corta que la de éstos, no haremos un análisis de correlación, pero dejamos apuntada el aparente paralelismo entre los dos procesos.

\section{FECUNDIDAD Y FAMILIAS}

Al ir agrupando los registros individuales en familias, a partir de los bautismos, confirmaciones y matrimonios, se han identificado 2.666 familias, en las que se integraban 6.118 hijos, de los cuales 68 eran mellizos o gemelos; fuera de la estructura familiar regular se han contado 368 hijos nacidos fuera de la institución familiar.

El cálculo del número de hijos por familia, dividiendo el total de hijos por el total de familias, incluidas las que no tienen hijos, nos da como resultado 2'29; por tanto, si a esta cifra le sumamos 2, correspondiente a los padres, tenemos el número de habitantes por familia, hogar o vecino, que sería, pues, de 4'29; valor que resaltamos por la importancia que tiene en los estudios históricos.

Habitantes por familia, hogar, o vecino: 4'29 
La distribución de las familias en relación al número de hijos la resumimos en la tabla siguiente:

\begin{tabular}{|c|c|c|}
\hline \multicolumn{3}{|c|}{ Composiciones familiares } \\
\hline Tipo de familia & N. ${ }^{\circ}$ de familias & Porcentaje \\
\hline - Familias sin hijos & 100 & $375 \%$ \\
\hline - Familias con 1 hijo & 1.049 & $3935 \%$ \\
\hline - Familias con 2 hijos & 593 & $22{ }^{\prime} 24 \%$ \\
\hline - Familias con 3 hijos & 390 & $14^{\prime} 63 \%$ \\
\hline - Familias con 4 hijos & 241 & $904 \%$ \\
\hline - Familias con 5 hijos & 128 & $4 ' 80 \%$ \\
\hline - Familias con 6 hijos & 81 & $3,04 \%$ \\
\hline - Familias con 7 hijos & 50 & $1 ' 88 \%$ \\
\hline - Familias con 8 hijos & 22 & $0 ' 83 \%$ \\
\hline - Familias con 9 hijos & 5 & $0,19 \%$ \\
\hline - Familias con 10 hijos & 3 & $0,11 \%$ \\
\hline - Familias con 11 hijos & 2 & $0,07 \%$ \\
\hline
\end{tabular}

Pero si del conjunto que hemos estudiado desglosamos los datos correspondientes a las familias cristianas y las moriscas, vemos lo siguiente:

\begin{tabular}{lcc}
\hline \multicolumn{3}{c}{ Distribución étnica de las familias } \\
\hline Grupo étnico & Cantidad & Porcentaje \\
\hline - Familias cristianas & 2.481 & $93^{\prime} 1 \%$ \\
6 & $6 \%$ \\
\hline - Familias moriscas & 185 & $92,7 \%$ \\
- Hijos de familias cristianas & 5.670 & $7{ }^{\prime} 3 \%$ \\
\hline
\end{tabular}

Hemos de hacer la salvedad de que somos conscientes de hacer una grosera diferenciación al separar a las familias de origen morisco de las cristianas, puesto que los datos que hemos manejado proceden de libros sacramentales cristianos, por lo que, teóricamente, todas eran cristianas; y sabemos que algunos se convirtieron sinceramente; si hubiéramos decido separarlas entre castellanas y moriscas, también habríamos incurrido en otro error, puesto que todas eran castellanas, en el sentido político de pertenencia a un reino. 
Volviendo a los datos antes expuestos, vemos que la familia morisca es, proporcionalmente, más prolífica que la cristiana, y los ratios respectivos son:

- Familia morisca: 2'42 hijos/familia

- Familia cristiana: 2'28 hijos/familia

y repercutiendo sobre cada composición familiar las personas de los progenitores, tenemos la siguiente composición de habitantes en los hogares:

- Familia morisca: 4'42 habitantes/hogar

- Familia cristiana: 4'28 habitantes/hogar

Valores que no están muy alejados de los que da $\mathrm{Nadal}^{8}$, cuando explica que para pasar de la cifra de vecinos a la de individuos, ha aplicado el multiplicador 4, pero cuando la presencia de moriscos era grande lo aumentaba a 4'3, e incluso, 4'5.

Para el conjunto de la población femenina emeritense, como parámetros demográficos, hemos calculado el tiempo que transcurría entre la celebración del matrimonio y el nacimiento del primer hijo, y luego el intervalo medio entre los hijos siguientes; estos valores son:

- Intervalo protogenésico: 2'18 años

- Intervalo intergenésico: 2'97 años

Es decir, la media de edad con la que la mujer alcanzaba la maternidad era de 24'85 años, mientras que para los varones la paternidad llegaba a los 29'28 años. En el cálculo del intervalo intergenésico se han tenido en cuenta los partos dobles, que se han contado lógicamente como uno sólo.

\footnotetext{
NADAL, J., "La población española durante los siglos xVi y xVii. Un balance a escala regional». Ob. cit, págs. 39 y 40.
} 